\section{La objeción de conciencia en la educación médica. Una propuesta para Chile}

\author{
SOFÍA P. SALAS
}

\section{Conscientious objectors in Chilean medical education}

The Chilean Law regulating the voluntary interruption of pregnancy, contemplates the possibility that health personnel may refrain from doing the procedure if they have stated that they are conscientious objectors (CO). There are numerous articles on the subject. However, the impact on medical training centers when a student or resident abstain from performing certain clinical procedures invoking $C O$, has seldom been analyzed. In this article, we explore the rights and duties of the CO students to perform an abortion or other clinical procedures for either religious or cultural reasons. Based on international experience, we recommend that all health care centers should have established and publicly known policies on this matter. Finally, we honor CO invoked by students, based on three general principles. First, the autonomy and moral integrity of the students should be respected. Second, an adequate ethical sensitivity is promoted. Third, it contributes to the necessary heterogeneity and diversity of students, promoting a desirable pluralism. However, certain interests and values, such as the well-being of patients, must be considered over and above accepting the $\mathrm{CO}$ requests.

(Rev Med Chile 2019; 147: 1067-1072)

Key words: Abortion, Legal; Conscience; Education, Medical; Students, Medical.
${ }^{1}$ Centro de Bioética, Facultad de Medicina, Clínica Alemana Universidad del Desarrollo. Santiago, Chile.

La autora declara no tener conflictos de interés.

Trabajo no obtuvo financiamiento externo.

Una versión extensa de este trabajo obtuvo el Premio de Ética 2018 otorgado por el Colegio Médico de Chile.

Recibido el 19 de noviembre de 2018, aceptado el 28 de agosto de 2019.

Correspondencia a:

Sofía P. Salas

Centro de Bioética, Facultad de Medicina, Clínica Alemana Universidad del Desarrollo. Avda. Las Condes 12.461, Torre 3, of 202. Santiago, Chile sofiasalas@udd.cl spsalas@gmail.com
$\mathrm{D}$ urante la aprobación de la Ley 20.130, que regula la interrupción del embarazo en tres causales, uno de los temas que concitó atención fue el de la objeción de conciencia $(\mathrm{OC})^{1}$. Su Reglamento garantiza la posibilidad de invocar la OC "al personal de salud al que corresponda desarrollar sus funciones al interior del pabellón quirúrgico durante la intervención"2. También permite que instituciones privadas de salud invoquen $\mathrm{OC}$, aun cuando estas no tienen conciencia moral, algo propio de los individuos, sino que poseen idearios valóricos, según los cuales rechazan entregar ciertas prestaciones $s^{3,4}$.

El objetivo del presente artículo es discutir los alcances de la OC en la educación médica, enfocándonos exclusivamente en la situación del estudiante de pre y postgrado que es objetor de conciencia. Por motivos de espacio, no nos referiremos respecto de centros objetores que deben formar a los profesionales de la salud que el país requiere.

\section{Conciencia moral, objeción de conciencia y profesión médica}

La conciencia moral es el juicio reflexivo del individuo, sobre lo que considera es un bien o un mal y que obliga a decidir entre posibles cursos de acción, respetando ese juicio. La OC es el 
derecho a negarse a acatar leyes o a realizar actos que contrarían convicciones éticas o morales del individuo; un ejemplo clásico es el de Muhammed Ali, quien rechazó alistarse en las FFAA de su país, debido a sus creencias religiosas contrarias al uso de la fuerza ${ }^{3,5}$. En el contexto médico, es la negativa a cumplir un cometido profesional exigido por las leyes impuestas por las autoridades legítimas, aduciendo para ello razones de conciencia ${ }^{4,6}$. Debemos recordar que la práctica de la medicina es fundamentalmente una actividad moral, que descansa en un pacto de confianza entre el médico y la persona enferma. Al convertirse en miembro de la profesión, el médico se compromete a defender los valores y obligaciones éticas fundamentales de esta, poniendo siempre al centro de su actividad el bienestar y auto-determinación de su paciente. $\mathrm{Al}$ mismo tiempo, los médicos son agentes morales con sus propias creencias y valores que, en ocasiones, entran en tensión con los del paciente ${ }^{7}$. El que la sociedad permita que algunos de sus miembros no cumplan con su deber profesional, significa necesariamente que terceros vean obstaculizado el acceso a una prestación de salud a la cual tienen legítimo derecho. Por lo tanto, la OC implica un conflicto ético entre los valores del objetor y los derechos de la persona que solicita una determinada prestación de salud.

La mayoría de los códigos de ética, incluyendo el del Colegio Médico de Chile, reconocen el derecho a la OC por parte del profesional sanitario ${ }^{8}$. Asimismo, la Asociación Médica Mundial, en su declaración sobre el término del embarazo por indicación médica, señala que "las convicciones del médico y del paciente deben ser respetadas" (Art. 6), reconociendo también que: "El médico tiene derecho a la objeción de conciencia para realizar un aborto, por lo que se puede retirar si asegura que un colega cualificado siga con la atención médica. En todo caso, el médico debe realizar los procedimientos necesarios para salvar la vida de la mujer y evitar graves lesiones a su salud" (Art. 8) ${ }^{9}$.

Existen diversos modelos para permitir la OC. Uno de estos es el de la protección absoluta, según el cual la conciencia prevalece ante cualquier otro interés, incluso respecto de los derechos de los propios pacientes; la aceptación de este modelo significa la anulación del Estado de Derecho. El otro modelo es el que considera que la conciencia es "privada", excluyendo las creencias y la conciencia del espacio público; este modelo termina anulando al ciudadano. Un modelo intermedio, que suscribimos, es el de la protección bilateral, que reconoce el derecho a la OC como algo excepcional, intentando armonizar y garantizar los derechos de las personas que solicitan la atención de salud y el de los profesionales que se abstienen de realizar un procedimiento legal, para defender su integridad moral ${ }^{10}$. Otras consideraciones respecto de los desafíos éticos de aceptar la OC han sido ampliamente desarrollados en Chile - $5,11,12^{2}$.

\section{Desafíos para la educación médica}

El que se respete el derecho a la OC por parte de agentes sanitarios y se permita que instituciones de salud invoquen la OC para no entregar prestaciones legales, impacta en la formación de los futuros profesionales de una doble manera. Por un lado, están los centros formadores que han invocado OC para no realizar una prestación, pero que deben garantizar que los estudiantes adquieran no solo una correcta formación teórica sino que las competencias prácticas requeridas para ejercer la medicina en una sociedad laica y pluralista. Por otra parte, está el centro formador, que debe aceptar que algunos de sus estudiantes rechacen realizar procedimientos que son considerados como competencias mínimas para certificar la especialidad, aspecto que desarrollaremos a continuación. Si bien nos centraremos principalmente en la práctica del aborto, debemos reconocer que el tema es más complejo en prácticas como las de la eutanasia, donde se debe enseñar la técnica adecuada para provocar la muerte del paciente, protocolo específico sin otro fin terapéutico.

\section{Dificultades con el estudiante/residente objetor}

En el año 2015, la Federación Internacional de Ginecología y Obstetricia abordó en profundidad el tema de la OC durante el entrenamiento clíni$\mathrm{Co}^{13}$, reforzando el concepto que el deber primario de los futuros especialistas es el de beneficiar y evitar dañar a las pacientes que ellos tratan, siendo la OC secundaria a este deber primario. En este contexto, se hace necesario que los residentes entreguen acceso oportuno a los servicios médicos requeridos, incluyendo toda la información disponible para que las mujeres tomen decisiones informadas, aun si ellos invocan OC. En consecuencia, solo se les puede eximir del deber de 
realizar la prestación, pero no de adquirir los conocimientos teóricos, dar consejería no directiva o hacer el diagnóstico adecuado, debiendo derivar para que otro profesional entregue la prestación ${ }^{14}$. Los residentes deben entregar información veraz, no sesgada según sus propias creencias y valores, $\mathrm{y}$ a su vez tienen el derecho a que se les respeten sus convicciones respecto de entregar o no entregar ciertos procedimientos, sin ser discriminados por ello. En encuestas de opinión realizadas a estudiantes de medicina en el Reino Unido y Finlandia, se observa amplia aceptación a la $\mathrm{OC}^{15,16}$, aunque esta aceptación varía según el contexto cultural y religioso de los estudiantes ${ }^{15,17}$.

Existen distintos modelos de entrenamiento para lograr las competencias necesarias. Uno es el "opt-out", según el cual el entrenamiento está disponible para todos, pero aquellos que invocan la OC no realizan la actividad práctica; con este modelo, los residentes refieren mayor adquisición de la competencia ${ }^{18}$. El otro modelo es el "opt-in", en el cual el entrenamiento no se entrega de manera rutinaria, dejando la responsabilidad al propio residente de querer realizar la actividad. Según algunos autores, este sistema refuerza una cultura de marginalidad en el servicio de aborto, otorgando menos posibilidades de adquirir las competencias. Por último, estarían aquellos centros formadores que no consideran entre sus objetivos el entrena- miento práctico para realizar abortos ${ }^{19}$, lo que sería preocupante para una especialidad que por ley está mandatada a entregar dicho servicio. No obstante, las técnicas de vaciamiento uterino deberán ser igualmente aprendidas con independencia de la OC, en casos de óbito fetal.

El que se permita a un estudiante eximirse de realizar una práctica contraria a su conciencia, debe ser considerado como algo excepcional y éste debe ser evaluado mediante actividades curriculares alternativas, que le permitan comprender el contexto relacionado con la práctica objetada ${ }^{16}$. El estudiante que invoca OC debe dar argumentos y realizar una actividad alternativa. Un reporte de la Asociación Médica Americana sugiere ciertos principios que deben ser cumplidos para permitir la OC durante el período de formación (Tabla 1$)^{14}$. Una negativa sistemática a la solicitud de eximirse de ciertas prácticas por motivos de conciencia, disminuye la sensibilidad ética de los estudiantes y puede propiciar que los estudiantes perciban que el éxito académico requiere de una suerte de "suspensión del juicio ético" ${ }^{14}$. El permitir la OC contribuye a mejorar el ambiente ético de todo el centro formador si estas solicitudes son consideradas de manera seria ${ }^{14}$. Asimismo, un rechazo sistemático a las solicitudes de los estudiantes podría disminuir la deseable diversidad a la que deben aspirar los centros formadores. Por último,

\section{Tabla 1. Requisitos para la eximición de actividades docentes por motivos de OC}

1. Las escuelas de medicina deben identificar los diversos conflictos que se originan entre la conciencia individual del médico y los deseos de los pacientes o las políticas de las instituciones de salud, como parte de las discusiones curriculares regulares sobre aspectos éticos y profesionales

2. Las escuelas de medicina deben tener mecanismos que permitan a los estudiantes ser excusados de actividades que violan sus creencias religiosas o éticas. Las escuelas deben definir y revisar periódicamente de qué tipo de actividades se puede eximir un estudiante al invocar OC, y qué alternativas curriculares son requeridas para los estudiantes que se eximen de cada actividad

3. Antes de matricularse, los futuros estudiantes deben ser informados de las políticas académicas relacionadas con la exención de actividades por motivos de conciencia

4. Deberían haber políticas escritas formales respecto de otorgar una dispensa por motivos de OC, incluyendo los procedimientos para obtenerla y el mecanismo para tratar asuntos de conciencia que no están cubiertos en las políticas formales

5. Las políticas relacionadas con las dispensas por motivos de OC deben ser aplicadas de manera consistente

6. Los estudiantes deben ser requeridos para aprender los contenidos básicos o los principios que subyacen los procedimientos o actividades que ellos pretenden eximirse de realizar. Cualquier excepción a este principio debe ser explícitamente descrito por la escuela

7. El cuidado del paciente no debe nunca ser comprometido si se permite a los estudiantes ser excusados de participar en una determinada actividad por motivos de conciencia

Adaptado y traducido por el autor desde el documento de la Asociación Médica Americana²3. 
el rechazo termina por socavar la integridad moral de los estudiantes, quienes se ven forzados a dejar de lado sus convicciones para poder progresar en sus estudios (para revisión, ver $\operatorname{Ref}^{14}$ ).

No obstante, como se expuso anteriormente, la aceptación de la OC invocada por estudiantes debe ser debidamente balanceada con el derecho de la paciente a obtener la prestación de salud que solicita. Esto es especialmente relevante si la solicitud de eximirse a realizar un procedimiento por motivos de conciencia se realiza sin tiempo suficiente para lograr que otro residente realice la prestación. Es necesario armonizar la solicitud de eximirse de una determinada práctica, con el análisis de aquellos requisitos curriculares que son considerados como mínimos y que incluso están contemplados en los procesos de acreditación y en los perfiles de egreso comúnmente aceptados ${ }^{14}$. Una de las prestaciones más controvertidas es precisamente la de realizar abortos, puesto que en esta se ponen en juego valores tan importantes como el concepto de dignidad humana, respeto a la autonomía de las mujeres y derecho a la vida del no nacido. Por estos motivos, en EEUU existe prohibición de discriminar a cualquier estudiante o residente que se niegue a recibir entrenamiento sobre el aborto o esterilizaciones, si esto se funda en motivos de conciencia ${ }^{14}$, y sería un error impedir que un estudiante objetor ingrese a la especialidad de G-O.

\section{Objeción de conciencia y multiculturalidad}

En un mundo globalizado y diverso, cada vez más los centros formadores serán interpelados por estudiantes que, aduciendo razones valóricas o religiosas, se niegan a realizar ciertos procedimientos que son considerados parte de las competencias clínicas necesarias para certificar la especialidad.

Una interesante discusión ha surgido respecto de si se puede certificar de manera adecuada las destrezas clínicas básicas que debe poseer cualquier médico en el caso de estudiantes que, por motivos de creencias religiosas y/o culturales, se niegan a realizar examen físico a personas de sexo opuesto, como ocurre con estudiantes musulmanes ${ }^{16}$. La religión musulmana considera que existe una cierta preferencia respecto del perfil del médico que debe examinar a un paciente (primero un musulmán del mismo sexo, luego un no-musulmán de igual sexo, un musulmán de distinto sexo y de no haber ninguno de los anteriores, un no musulmán de distinto sexo), aunque permite desviarse de la norma en caso de emergencia ${ }^{20}$. La realización de un examen físico completo a pacientes de ambos sexos es considerada como una práctica clínica esencial, por lo que no sería admisible que se aceptara que un futuro médico no tuviera las destrezas necesarias para examinar a hombres y mujeres. Concordando con argumentos de otros autores, es necesario que los futuros profesionales distingan lo que es "tocar" como algo propio del acto médico, carente de toda significación erótica o sexual, de otras connotaciones del contacto físico ${ }^{16}$. Así, pareciera no haber otra alternativa que negar el invocar OC respecto de ciertos actos que son propios de la certificación de un médico, especialmente si no existen formas alternativas de adquirir la competencia ${ }^{14}$. Una situación parecida ha sido descrita en relación a estudiantes que se niegan a aprender respecto de problemas relacionados con el abuso de alcohol y drogas o de enfermedades de trasmisión sexual, por considerar que estos temas son contrarios a sus creencias religiosas ${ }^{14}$. Sin este conocimiento teórico, no estarían en condiciones de plantear diagnósticos diferenciales, indicar exámenes de laboratorio o realizar indicaciones terapéuticas, por lo que su solicitud debiese ser rechazada.

Aunque no es tan frecuente en nuestro país, algunos estudiantes han solicitado que se les exima de realizar turnos en ciertos días y horarios, por motivos religiosos. Las Escuelas de Medicina han realizado propuestas para flexibilizar los requisitos de asistencia y formas de recuperar las actividades prácticas, que idealmente deben ser planificadas, para lo cual se solicita que los estudiantes envíen por escrito sus requerimientos. Dependerá de las circunstancias específicas si se logra modificar todas las actividades curriculares, pero atendiendo a la libertad de culto ${ }^{21}$, se hace necesario que se hagan los máximos esfuerzos por permitir que los estudiantes vivan su fe sin ser penalizados académicamente por aquello.

\section{Recomendaciones}

La experiencia internacional en el manejo de estudiantes que invocan OC respecto de ciertas prácticas clínicas, sugiere fuertemente que es deseable que cada centro tenga una política específica 
sobre los derechos y deberes del alumno objetor, que sea conocida de antemano ${ }^{22}$. Estas normas deben incluir los procedimientos necesarios para que la petición sea estudiada y las formas de resolver potenciales conflictos. Si bien es deseable que en un mundo diverso se respeten las creencias y valores de toda la comunidad académica, en ocasiones no podrá acogerse la petición, si esto es demasiado gravoso para la entidad. Un ejemplo es la negativa a realizar actividades académicas en día sábado; en algunos casos se podrá aceptar el requerimiento, pero no siempre se podrá programar una actividad especial en otro día, como suele suceder con los exámenes prácticos estructurados. La Asociación Médica Americana hace un llamado a las escuelas de medicina a identificar aquellas actividades que califican para hacer OC y los mecanismos formales para obtener dicha dispensa ${ }^{23}$. Dado que los estudiantes, especialmente durante el pregrado, siguen formándose moralmente, las Escuelas pueden aceptar cambios fundamentados de la OC durante el desarrollo de la carrera.

\section{Conclusiones}

Existen numerosas razones por las cuales un centro formador deba permitir que sus estudiantes invoquen razones de conciencia para solicitar no realizar un determinado procedimiento. En primer lugar, el prohibir la OC de los estudiantes podría amenazar con pasar a llevar su autonomía e integridad moral. En segundo lugar, el respeto a la OC promueve una adecuada sensibilidad ética y mejora así todo el proceso de reflexión y deliberación requerido para fundamentar su exclusión de una determinada práctica. Finalmente, un rechazo a la solicitud terminaría disminuyendo la necesaria heterogeneidad y diversidad estudiantil, puesto que los estudiantes objetores evitarían postular al centro que no tolera la $\mathrm{OC}^{22}$. No obstante, existen ciertos intereses y valores que deben ser considerados cuando se contempla la posibilidad de acceder a la solicitud. Entre estos están el establecer ciertas competencias claves, que deben ser adquiridas por todos los estudiantes; considerar cómo el currículo local se puede adaptar a estas peticiones; no discriminar a estudiantes o residentes objetores; y el impacto que esto pudiese tener en los pacientes, en otros estudiantes y en el cuerpo académico.

En la medida que se promuevan políticas de respeto a la OC conocidas por todos, incluyendo futuros postulantes, se podrán tomar decisiones informadas respecto de la elección de lugares donde realizar la formación clínica y la elección de especialidad. En este contexto, nos parece que centros formadores con políticas intolerantes respecto de estudiantes objetores obstaculizarán el crear un ambiente académico diverso y pluralista lo que, a la larga, terminará desfavoreciendo a futuros pacientes.

\section{Referencias}

1. Ley 20.130. Regula la despenalización de la interrupción voluntaria del embarazo en tres causales. Publicada el 23.09.2017, MINSAL, Chile. Disponible en http://bcn. cl/222kz (Fecha de acceso 25 de octubre de 2018).

2. Aprueba reglamento para ejercer objeción de conciencia según lo dispuesto en el artículo 119 Ter del Código Sanitario. Publicado en el Diario Oficial de la República de Chile, Núm 42.187, de fecha 23 de octubre de 2018.

3. Beca JP, Astete CA. Objeción de conciencia en la práctica médica. Rev Med Chile 2015; 143 (4): 493-8.

4. Salas SP, Besio M, Borquez Estefo G, Salinas RA, Valenzuela CY, Micolich C, et al. [Position paper from the Department of Ethics of the Chilean College of Physicians about conscientious objection]. Rev Med Chil 2016; 144 (3): 382-7.

5. Besio M. Objeción de conciencia, Profesión médica y Proyecto sobre despenalización del aborto en Chile. Rev Med Chile 2016; 144: 377-81.

6. Morales-Guzmán-Barrón R. Consciousness objection and the medical student. Rev Soc Peru Med Interna 2008; 21 (2): 78-81.

7. Mccormick PW. Report 1 of the Council on ethical and judicial affairs (CEJA Report 1-I-14). Physician Exercise of Conscience. Disponible en https://http://www.amaassn.org/sites/default/files/media-browser/public/aboutama/councils/Council Reports/council-on-ethics-andjudicial-affairs/i14-ceja-physician-exercise-conscience. pdf (Fecha de acceso 27 de octubre de 2018).

8. Colegio Médico de Chile AG, Código de Ética 2011: Editado e impreso por Ediciones Tierra Mía Ltda. Santiago de Chile.

9. Asociación Médica Mundial. Declaración sobre el término del embarazo por indicación médica. Adoptada por la 24a Asamblea Médica Mundial Oslo, Noruega, agosto 1970, y enmendada por la 35a Asamblea Médica Mundial Venecia, Italia, octubre 1983, la 57a Asamblea General de la AMM, Pilanesberg, Sudáfrica, octubre 
2006, y la 69a Asamblea General de la AMM, Reykjavik, Islandia, octubre 2018.

10. Couceiro A, Seoane JA, Hernando P. [Conscientious objection in the clinical setting. A proposal for its appropriate use]. Rev Calid Asist 2011; 26 (3): 188-93.

11. Montero Vega A. La objeción de conciencia de los profesionales y su relación con la atención en salud sexual y reproductiva de adolescentes en Santiago de Chile. Acta bioeth 2014; 20: 197-206.

12. Casas L. Invoking conscientious objection in reproductive health care: evolving issues in Peru, Mexico and Chile. Reprod Health Matters 2009; 17 (34): 78-87.

13. Figo Committee For The Ethical Aspects Of Human Reproduction And Women's Health; International Federation of Gynecology and Obstetrics. Ethical guidelines on conscientious objection in training. Int J Gynaecol Obstet 2015; 128 (1): 89-90.

14. Wicclair MR. Conscience-based exemptions for medical students. Camb Q Healthc Ethics 2010; 19 (1): 38-50.

15. Nordstrand SJ, Nordstrand MA, Nortvedt P, Magelssen M. Medical students' attitudes towards conscientious objection: a survey. J Med Ethics 2014; 40 (9): 609-12.

16. Card RF. Is there no alternative? Conscientious objection by medical students. J Med Ethics 2012; 38 (10): 602-4.
17. Strickland SL. Conscientious objection in medical students: a questionnaire survey. J Med Ethics 2012; 38 (1): 22-5.

18. Turk JK, Preskill F, Landy U, Rocca CH, Steinauer JE. Availability and characteristics of abortion training in US ob-gyn residency programs: a national survey. Contraception 2014; 89 (4): 271-7.

19. Committee on Health Care for Underserved Women. ACOG Committee opinion no. 612: Abortion training and education. Obstet Gynecol 2014; 124 (5): 1055-9.

20. Padela AI, Rodríguez del Pozo P. Muslim patients and cross-gender interactions in medicine: an Islamic bioethical perspective. J Med Ethics 2011; 37(1):40-4.

21. Ley 19.638. Ministerio del Interior. Establece normas sobre la constitución jurídica de las iglesias y organizaciones religiosas. Promulgada el 01-10-1999. Disponible en http://www.leychile.cl/N?i=145268\&f=1999-10-14\&p= (Fecha de acceso 30 de octubre de 2018).

22. Wicclair M. Conscientious Objection in Health Care: An Ethical Analysis. Cambridge University Press, 2011. ISBN 1139500198. Disponible en https://books.google. cl/.p 199-202. 2011.

23. American Medical Association Policy H-295.896, 2018. Conscience Clause: Final Report. Disponible en https:// policysearch.ama-assn.org/policyfinder/detail/Conscience\%20Clause?uri=\%2FAMADoc\%2FHOD.xml-0-2195. xml (Fecha de acceso 25 de octubre de 2018). 